\title{
Mitogen-Activated Protein Kinase Phosphatase-2 Deletion Impairs Synaptic Plasticity and Hippocampal-Dependent Memory
}

\author{
Nor Zaihana Abdul Rahman, ${ }^{1}$ Sam M. Greenwood, ${ }^{1}$ Ros R. Brett, ${ }^{1}$ Kyoko Tossell, ${ }^{2}$ Mark A. Ungless, ${ }^{2}$ Robin Plevin, ${ }^{1}$ \\ and Trevor J. Bushell ${ }^{1}$ \\ ${ }^{1}$ Strathclyde Institute of Pharmacy and Biomedical Sciences, University of Strathclyde, Glasgow G4 0RE, United Kingdom, and ${ }^{2}$ Medical Research Council \\ Clinical Sciences Centre, Imperial College London, Hammersmith Hospital, Du Cane Road, London W12 0NN, United Kingdom
}

Mitogen-activated protein kinases (MAPKs) regulate brain function and their dysfunction is implicated in a number of brain disorders, including Alzheimer's disease. Thus, there is great interest in understanding the signaling systems that control MAPK function. One family of proteins that contribute to this process, the mitogen-activated protein kinase phosphatases (MKPs), directly inactivate MAPKs through dephosphorylation. Recent studies have identified novel functions of MKPs in development, the immune system, and cancer. However, a significant gap in our knowledge remains in relation to their role in brain functioning. Here, using transgenic mice where the Dusp4 gene encoding MKP-2 has been knocked out (MKP-2 $2^{-1-}$ mice), we show that long-term potentiation is impaired in MKP-2 $2^{-/-}$mice compared with MKP- $2^{+/+}$controls whereas neuronal excitability, evoked synaptic transmission, and paired-pulse facilitation remain unaltered. Furthermore, spontaneous EPSC (sEPSC) frequency was increased in acute slices and primary hippocampal cultures prepared from MKP-2 ${ }^{-1-}$ mice with no effect on EPSC amplitude observed. An increase in synapse number was evident in primary hippocampal cultures, which may account for the increase in sEPSC frequency. In addition, no change in ERK activity was detected in both brain tissue and primary hippocampal cultures, suggesting that the effects of MKP-2 deletion were MAPK independent. Consistent with these alterations in hippocampal function, MKP- $2^{-1-}$ mice show deficits in spatial reference and working memory when investigated using the Morris water maze. These data show that MKP-2 plays a role in regulating hippocampal function and that this effect may be independent of MAPK signaling.

Key words: hippocampal-dependent memory; MAPK; MKP-2; sEPSC; synaptic plasticity

\section{Significance Statement}

Recently, there has been significant focus on proteins that control mitogen-activated protein kinases' (MAPKs) function, namely the mitogen-activated protein kinase phosphatases (MKPs). Recent studies have revealed novel roles for a specific MKP, MKP-2, in the immune system and cancer. In the present study, we focus on MKP-2 to determine its role in neuronal function. Using transgenic mice where the Dusp4 gene encoding MKP-2 has been knocked out, we use a number of techniques to reveal that MKP-2 deletion increases spontaneous neurotransmitter release, impairs the induction of synaptic plasticity, and induces deficits in hippocampal-dependent memory. These findings provide a new insight into role that MKP-2 plays in regulation hippocampal function and that this may be independent of MAPK signaling.

\section{Introduction}

Cellular maintenance and survival is dependent on multiple cell signaling pathways, including mitogen-activated protein kinase (MAPK) signaling. The MAPK family consists of

\section{Received Oct. 20, 2015; revised Nov. 30, 2015; accepted Dec. 8, 2015}

Author contributions: N.Z.A.R., S.M.G., R.R.B., K.T., M.A.U., and T.J.B. designed research; N.Z.A.R., S.M.G., R.R.B., and K.T. performed research; R.P. contributed unpublished reagents/analytic tools; N.Z.A.R., S.M.G., R.R.B., K.T., M.A.U., and T.J.B. analyzed data; N.Z.A.R., S.M.G., R.R.B., K.T., M.A.U., R.P., and T.J.B. wrote the paper.

N.Z.A.R. was funded by a scholarship from University Sains Islam Malaysia and the Ministry of Higher Education, Malaysia; the immunohistochemical research was supported by Grant MC-A654-5QB70 from the UK Medical Research Council to M.A.U. the extracellular signal-related kinase (ERK), the c-jun N-terminal kinase (JNK), and p38 MAPK (Cuevas et al., 2007;

The authors declare no competing financial interests.

This article is freely available online through the J Neurosci Author Open Choice option.

Correspondence should be addressed to Dr Trevor Bushell, University of Strathclyde, 161 Cathedral Street, Glasgow G4 ORE, UK. E-mail: trevor.bushell@strath.ac.uk.

D0I:10.1523/JNEUROSCI.3825-15.2016

Copyright $\odot 2016$ Abdul Rahman et al.

This is an Open Access article distributed under the terms of the Creative Commons Attribution License Creative Commons Attribution 4.0 International, which permits unrestricted use, distribution and reproduction in any medium provided that the original work is properly attributed. 
Keshet and Seger, 2010). MAPK proteins are expressed in numerous cell types within the CNS where they play an important role in the regulation of synaptic transmission and plasticity (Thomas and Huganir, 2004; Samuels et al., 2009). In addition, MAPKs have a role in modulating learning and memory (Ciccarelli and Giustetto, 2014) and their dysfunction has also been implicated in a range of CNS disorders including Alzheimer's disease, multiple sclerosis, and Parkinson's disease (Samuels et al., 2009; Yasuda et al., 2011; Krementsov et al., 2013).

The kinetics of MAPK signaling are directly regulated through the activation and/or expression of a family of dualspecificity phosphatases (DUSPs), the mitogen-activated protein kinase phosphatases (MKPs), that dephosphorylate MAPKs and thus inhibit their activity (Caunt and Keyse, 2013). MKPs have recently been shown to play key roles in development, immunity, and cancer (Lawan et al., 2012; Wancket et al., 2012). However, our knowledge regarding MKP function in the brain is extremely limited. Although studies support a role for the prototypic MKP, Dusp1 (MKP1), in axonal branching (Jeanneteau et al., 2010) and depression (Duric et al., 2010), the potential role of Dusp4 (MKP-2) function in the CNS has been largely neglected. To elucidate the potential role of MKP-2 in brain function, given the absence of specific MKP-2 inhibitors, here we assess hippocampal neuronal excitability and synaptic plasticity and hippocampal-dependent behavior in MKP-2 $2^{-1-}$ mice.

\section{Materials and Methods}

Animals

Heterozygote MKP-2 mice backcrossed on a C57BL/6J background generated homozygous and wild-type littermates that were used for breeding colonies (Al-Mutairi et al., 2010). All breeding and experimentation were in accordance with UK legislation [Animals (Scientific Procedures) Act 1986] and with approval by the University of Strathclyde Ethics Committee.

\section{Tissue preparation}

Primary hippocampal cultures were prepared and maintained from 1- to 2-d-old MKP-2 $2^{+/+}$or MKP-2 ${ }^{-/-}$pups as described previously (Gan et al., 2011). Parasagittal hippocampal slices $(400 \mu \mathrm{m})$ were prepared from male mice (19- to 24-d-old) as previously described (Gan et al., 2011) with slices initially incubated at $35^{\circ} \mathrm{C}$ for $30 \mathrm{~min}$, and then allowed to equilibrate for $1 \mathrm{~h}$ at room temperature before use.

\section{Electrophysiology}

Cultured neurones. Hippocampal cultures were perfused at a flow rate at 1-2 $\mathrm{ml} \mathrm{min}^{-1}$ with HEPES-buffered saline composed of the following (in mM): $\mathrm{NaCl} 140, \mathrm{KCl} 5, \mathrm{MgCl}_{2} 2, \mathrm{CaCl}_{2}$ 2, HEPES 10, D-glucose 10, $\mathrm{pH}$ 7.4, $310 \mathrm{mOsm}$. Fire-polished glass microelectrodes ( $3-5 \mathrm{M} \Omega$ ) were filled with intracellular solution containing the following (in $\mathrm{mM}$ ): $\mathrm{KMeSO}_{4}$ 130, KCl 20, Mg-ATP 4, Na-GTP 0.3, EGTA 0.3, HEPES 10, pH 7.2, 290 mOsm. sEPSCs were recorded at $-70 \mathrm{mV}$ for 5 min periods at room temperature in voltage-clamp mode using WinEDR software (J. Dempster, University of Strathclyde, UK), digitized at $5 \mathrm{kHz}$, and analyzed off-line using Mini analysis software (Synaptosoft). All experiments were performed on at least three independent cultures.

Acute slices. Slices were transferred into a submerged recording chamber maintained at $30^{\circ} \mathrm{C}$ and continuously perfused with oxygenated

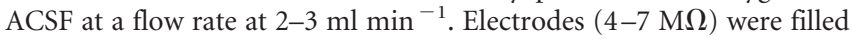
with an internal solution containing the following (in $\mathrm{mM}$ ): $130 \mathrm{Cs} / \mathrm{K}$ $\mathrm{MeSO}_{4}, 8 \mathrm{NaCl}, 4 \mathrm{Mg}$-ATP, $0.3 \mathrm{Na}$-GTP, 0.5 EGTA, 10 HEPES, pH 7.2, $290 \mathrm{mOsm}$. Whole-cell patch-clamp recordings were made from CA1 pyramidal neurons using WinWCP or WinEDR software (J. Dempster, University of Strathclyde, UK). For current-voltage relationships $\left(\mathrm{K}^{+}\right.$ internal), current injections at 10 s intervals was used. For synaptic transmission $\left(\mathrm{Cs}^{+}\right.$internal), the Schaffer collateral-commissural pathway was stimulated with a bipolar electrode $(0.033 \mathrm{~Hz})$ with responses set to $50 \%$ of maximum. For paired-pulse facilitation (PPF) experiments, interstimulus intervals (ISI, $50-500 \mathrm{~ms}$ ) were used with long-term potentiation (LTP) being induced using a pairing protocol (100 stimuli, $1 \mathrm{~Hz}$, $V_{\mathrm{H}}=0 \mathrm{mV}$ ) with data digitized at $10 \mathrm{kHz}$ and analyzed off-line using WinWCP. sEPSCs were recorded at $-70 \mathrm{mV}$ for 5 min periods using WinEDR, digitized at $5 \mathrm{kHz}$, and analyzed off-line using Mini analysis software (Synaptosoft). For all slice experiments, $n$ is equal to one slice per animal with the experimenter being blind to genotype.

\section{Immunostaining}

Cultures. Cultures were fixed in ice-cold 4\% paraformaldehyde (PFA; 10 $\mathrm{min}$ ) followed by ice-cold methanol (100\%, $10 \mathrm{~min})$, and then permeabilized with $0.01 \%$ Triton-X (in PBS, $10 \mathrm{~min}$ ), followed by incubation in a blocking solution [ $5 \%$ fetal bovine serum, $1 \%$ bovine serum albumin (BSA) in PBS, $1 \mathrm{~h}$ ]. Primary antibodies [anti- $\beta$ III-tubulin (1:500), Sigma-Aldrich no. T8578; anti-synaptophysin (1:500), Millipore no. MAB368; pERK (1:500) Cell Signaling Technology no. 9106; total ERK (1:500), Santa Cruz Biotechnology no. sc-514302] were applied directly to the coverslip and incubated overnight at $4^{\circ} \mathrm{C}$. Cultures were then washed and incubated $(1 \mathrm{~h})$ with the appropriate fluorescent secondary antibody (1:200, AlexaFluor 488 no. A10538, AlexaFluor 555 no. A-31572, Life Sciences) diluted in blocking buffer. Cultures were then washed and images acquired using epifluorescent microscopy. Synapse number and fluorescent intensity was measured using NIH ImageJ software with all data analyzed from at least three separate cultures.

Slices. Male mice (10- to 12-weeks-old) were perfused transcardially with $4 \%$ PFA. Brains were removed and postfixed in 4\% PFA (1 h), washed with PBS, and followed by cryoprotection in 30\% sucrose. Coronal brain slices $(70 \mu \mathrm{m})$ were prepared using a cryostat (Leica). Slices were rinsed with PBS and agitated in PBS with $0.5 \%$ Triton $\mathrm{X}-100$ (15 min). Free-floating slices were then pre-blocked $(1 \mathrm{~h})$ at room temperature in PBS with $0.2 \%$ Triton X-100, 6\% normal donkey serum (NDS; Jackson ImmunoResearch), and incubated overnight at $4^{\circ} \mathrm{C}$ with mouse monoclonal NeuN antibody (1:1000, Abcam no. ab104224) with $0.2 \%$ Triton X-100 and $2 \%$ NDS. Slices were rinsed in PBS ( $1 \mathrm{~h})$ and incubated $(2 \mathrm{~h})$ with donkey anti-mouse Cy3 (1:1000, Jackson ImmunoResearch, no. 715-165-150) and DAPI (1: 1000, Santa Cruz Biotechnology) with 2\% Triton X-100 and 2\% NDS. After subsequent PBS rinses, slices were mounted on slides using Fluoromount Aqueous Mounting Medium (Sigma-Aldrich) and visualized using a Leica SP5 confocal laser-scanning microscope.

\section{Western blot}

Total protein from the cerebellum, cortex and hippocampus from male mice (19- to 24-d-old) was used for Western blot analysis. Briefly, protein samples $(10 \mu \mathrm{g})$ were loaded in $10 \%$ SDS polyacrylamide gels, subjected to electrophoresis, and blotted onto a nitrocellulose sheet with nonspecific binding was blocked using a NaTT buffer containing $4 \%$ BSA ( $2 \mathrm{~h})$. Blots were then incubated overnight with specific primary antibodies for p-ERK (1:500, Santa Cruz Biotechnology, no. sc-32577) prepared in $0.4 \%$ BSA/NaTT, washed with NaTT buffer before incubating with horse-radish peroxidaseconjugated anti-mouse IgG secondary antibody $(1.5 \mathrm{~h})$. Blots were then washed $(2 \mathrm{~h})$ and developed. After detection of phosphorspecific bands, blots were stripped and re-probed with total ERK antibody (1:500, Santa Cruz Biotechnology, no. sc-514302). Bands were quantified using densitometry with Scion Image software with values expressed as percentage of p-ERK over total ERK.

\section{Behavioral testing}

MKP-2 ${ }^{-1-}$ and MKP-2 ${ }^{+/+}$mice $(n=22-24)$ were tested in the following order: open field test (OF), elevated plus-maze (EPM), and Morris water maze (MWM) with mice videoed and tracked by Ethovision software (Noldus).

OF: distance moved in a 40-cm-square open field, and center square $(14 \mathrm{~cm})$ entries and time were recorded for $10 \mathrm{~min}$. EPM: the number of open and closed arm entries during $10 \mathrm{~min}$ in a plus- 
a

C

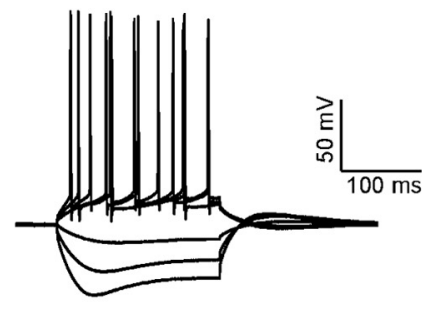

b

d
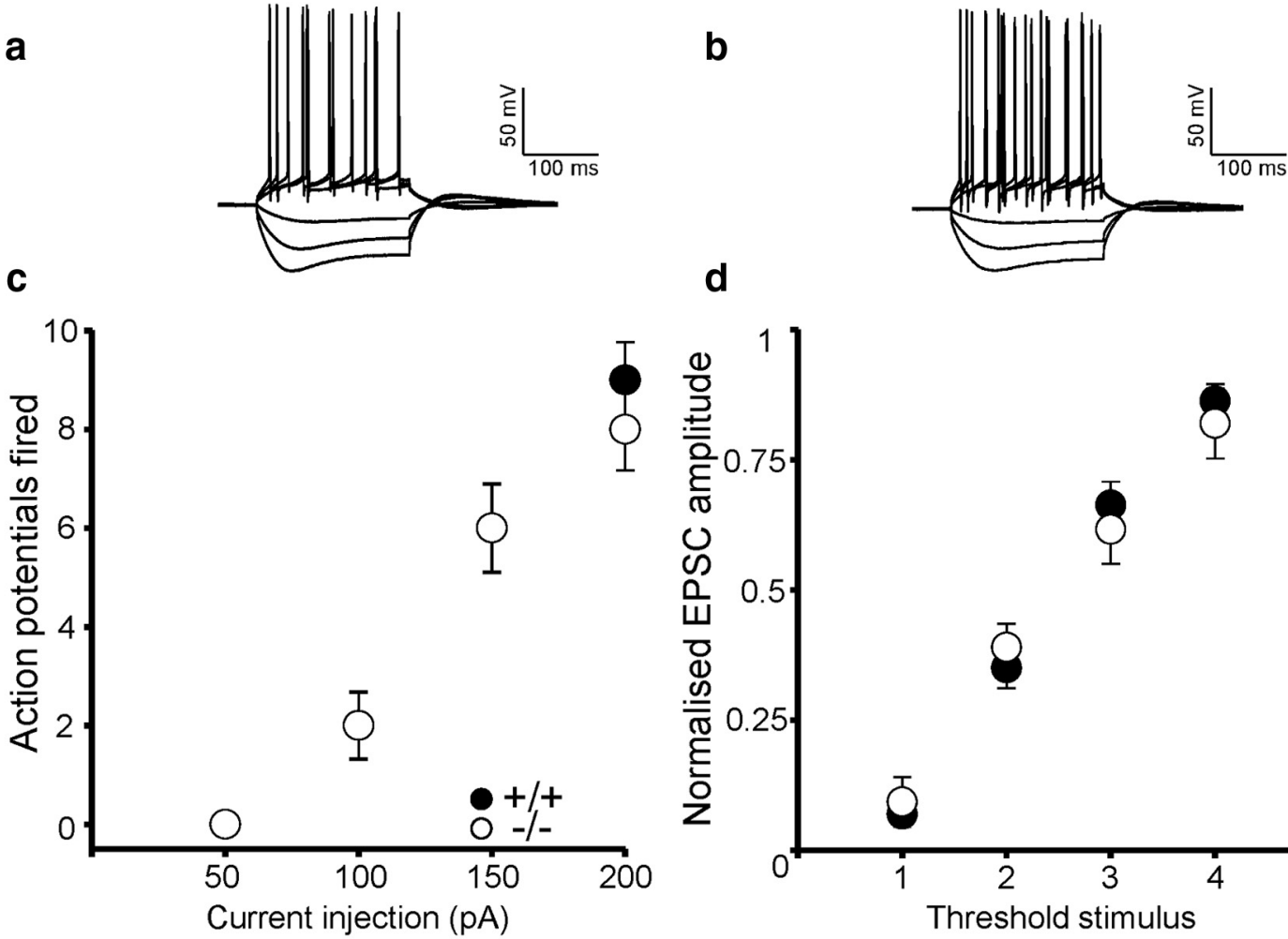

e
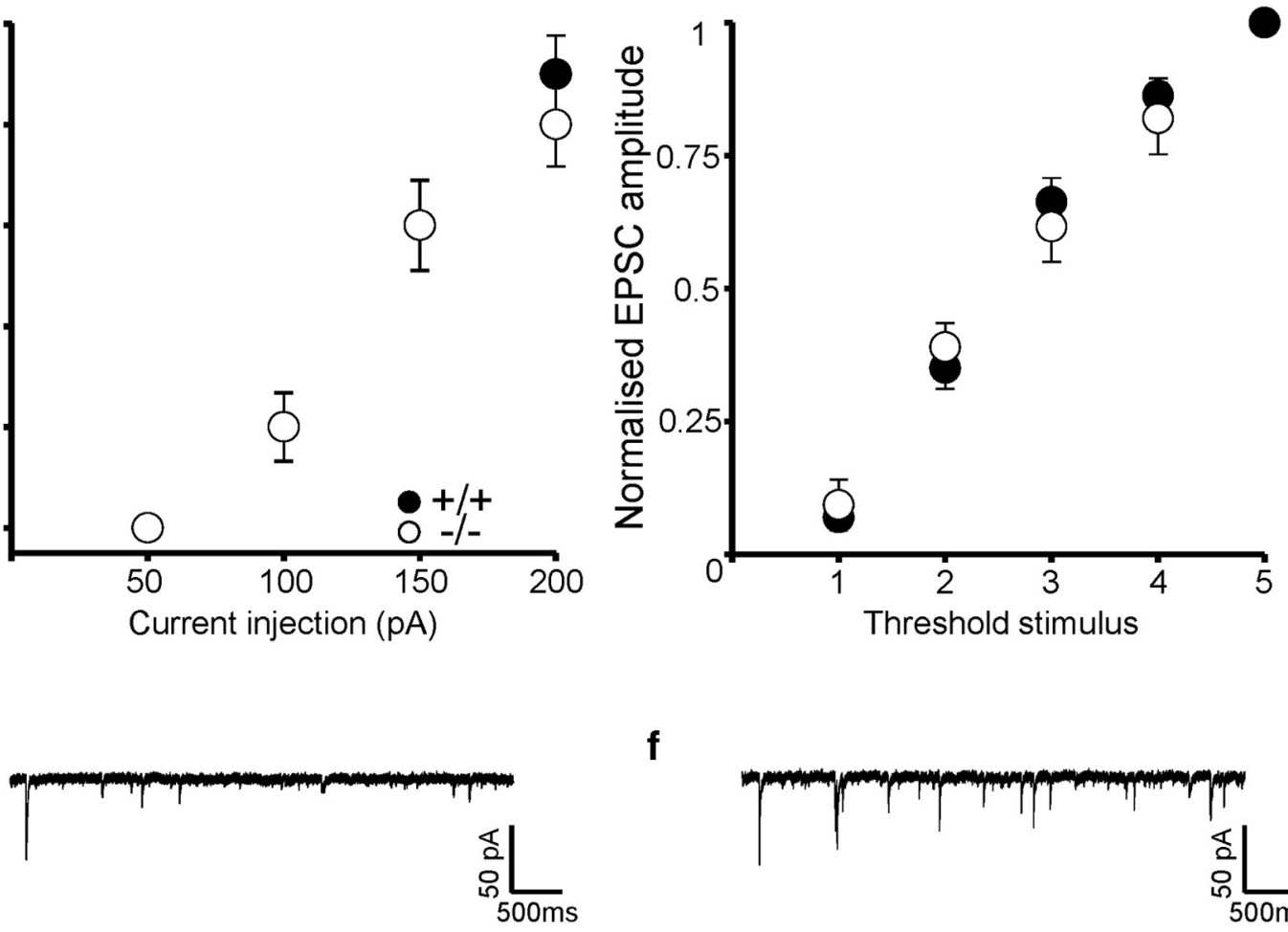

f

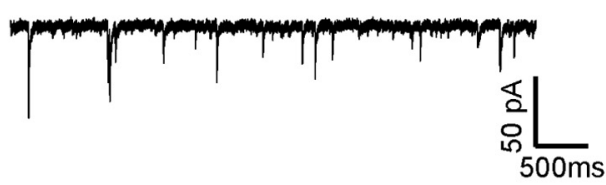

9

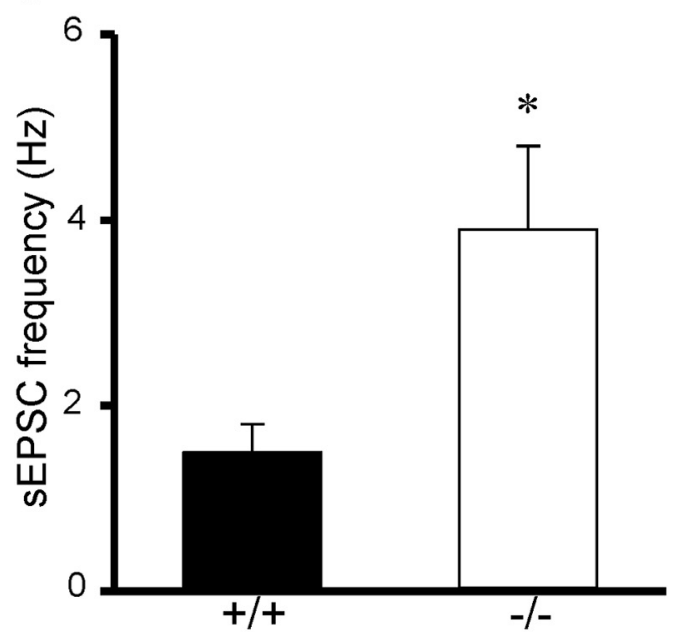

h

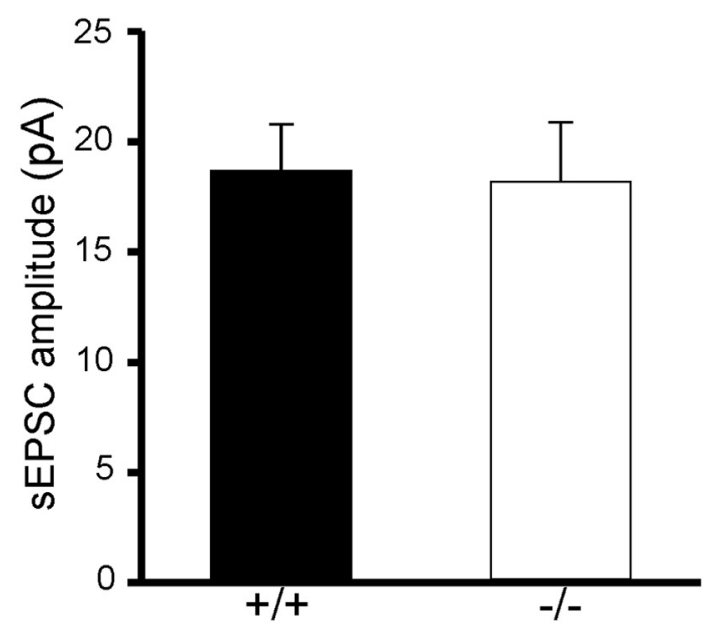

Figure 1. Increased sEPSC frequency but normal neuronal excitability and synaptic transmission in MKP-2 $2^{-1-}$ hippocampal slices. $\boldsymbol{a}, \boldsymbol{b}$, Representative traces from MKP-2 $2^{+/+}$and MKP-2 $2^{-/-}$ slices respectively. $\boldsymbol{c}$, No alteration in AP firing or (d) evoked transmission in MKP- $2^{-/-}$slices. $\boldsymbol{e}, \boldsymbol{f}$, Representative SEPSC traces from MKP-2 ${ }^{+/+}$and MKP-2 ${ }^{-/-}$slices respectively. $\boldsymbol{g}$, Increased SEPSC frequency is evident in MKP $-2^{-1-}$ slices, whereas $(\boldsymbol{h})$ sEPSC amplitude is unaltered. ${ }^{*} p<0.05$.

shaped maze with two open $(30 \times 5 \mathrm{~cm})$ and two closed arms $(30 \times 5$ $\mathrm{cm}, 15 \mathrm{~cm}$ walls) elevated $70 \mathrm{~cm}$ from the floor were recorded and percentage open arm entries and time calculated. MWM: mice were tested in a $98-\mathrm{cm}$-diameter maze containing water at $21^{\circ} \mathrm{C}$ with a transparent 10 -cm-diameter submerged platform, in a room with extra-maze cues. Reference memory: three to four times daily for $5 \mathrm{~d}$, mice were released at one of four randomly varied points and swam until they located the platform. Platform location remained constant for each mouse. Path-length and latency to reach the platform were calculated as the mean of each day's trials. On the final trial of day 5 , the platform was removed and mice allowed to swim for $60 \mathrm{~s}$ (probe test). Time spent in the quadrant of the previous location of the 
a

C

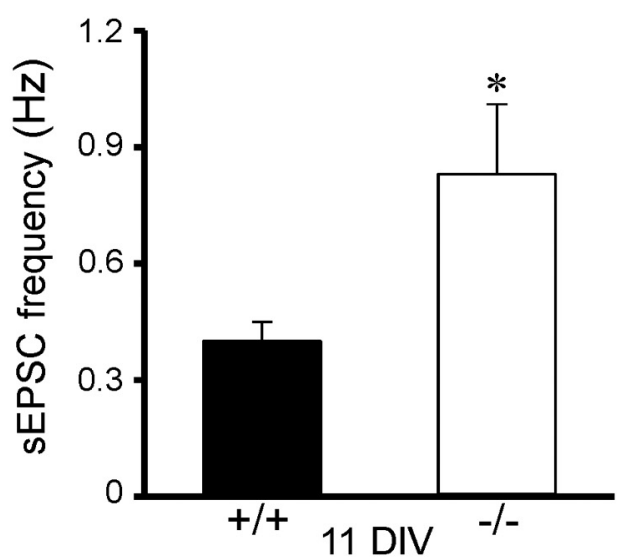

e

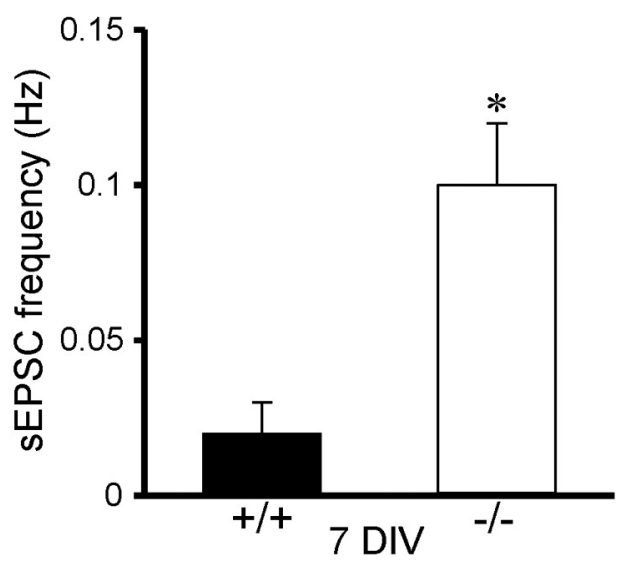

g

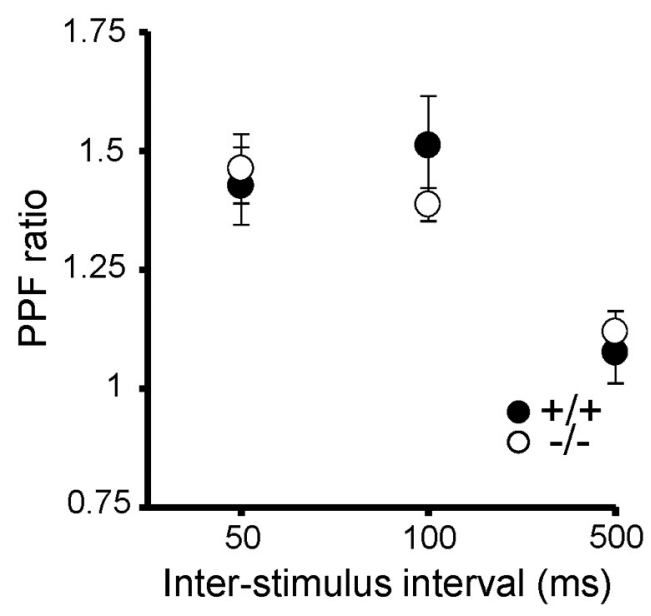

b

d
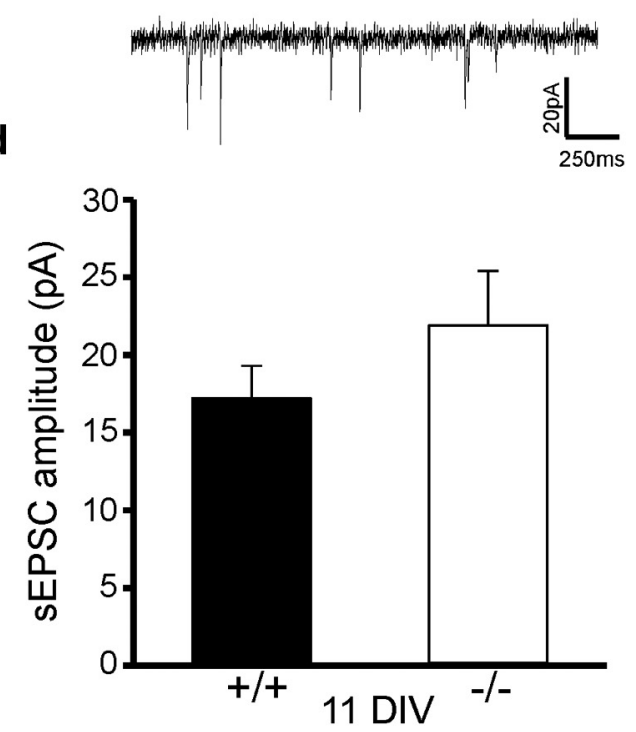

f

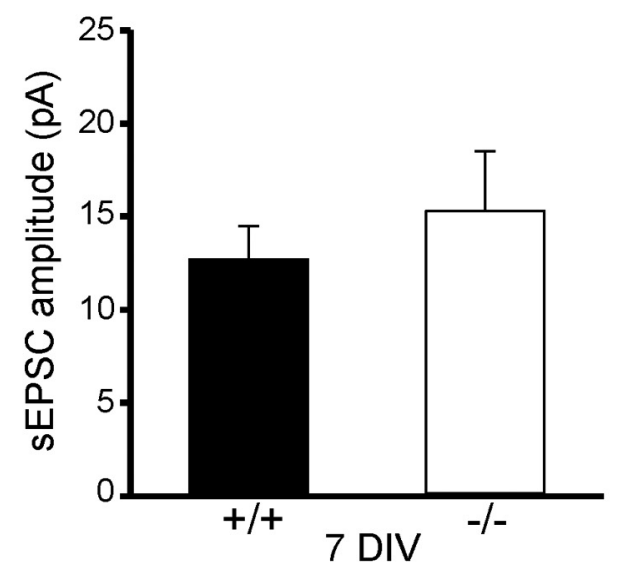

h

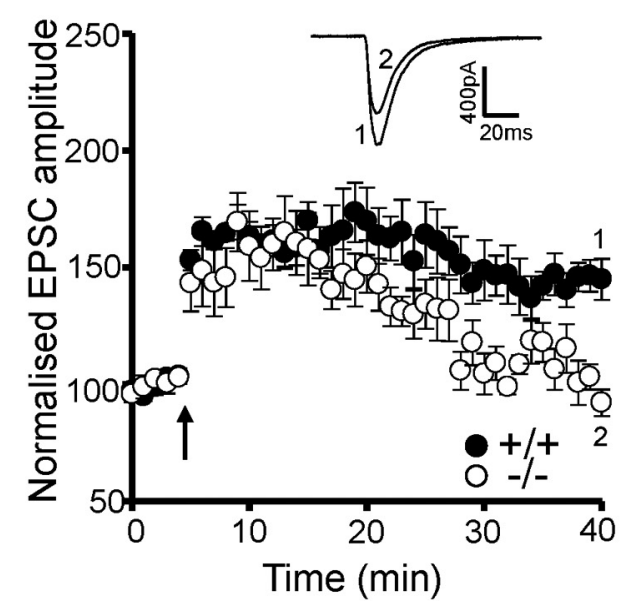

Figure 2. Increased sEPSC frequency in MKP-2 ${ }^{-/-}$cultures but impaired LTP in MKP $-2^{-/-}$slices. $\boldsymbol{a}, \boldsymbol{b}$, Representative traces of sEPSCs in MKP-2 $2^{+/+}$and MKP-2 $2^{-/-}$mice cultures (11 DIV) respectively. $\boldsymbol{c}, \boldsymbol{d}$, Increased SEPSC frequency but not amplitude in 11 DIV MKP- $2^{-1-}$ primary hippocampal cultures. $\boldsymbol{e}, \boldsymbol{f}$, Increased sEPSC frequency but not amplitude in 7 DIV MKP-2 ${ }^{-1-}$ primary hippocampal cultures. $\boldsymbol{g}$, PPF is unaltered in MKP-2 ${ }^{-/-}$slices, whereas (h) LTP is impaired compared with MKP-2 ${ }^{+/+}$controls. ${ }^{*} p<0.05$.

platform (target quadrant) and the opposite quadrant was recorded. Working memory: $3 \mathrm{~d}$ later, mice were tested with a daily changing platform position ( $7 \mathrm{~cm}$ platform to increase difficulty) for $3 \mathrm{~d}$. Each day, mice were allowed up to seven trials and given a score equaling the number of trials to reach criterion (successful location in $\leq 10 \mathrm{~s}$ twice consecutively). If criterion was not reached by the seventh trial, a score of 8 was given if it would be possible to reach criterion on the next trial, otherwise a score of 9 . 
Statistics

All data are expressed as mean \pm SEM. Data were compared by paired or unpaired Student's $t$ tests, one-way ANOVA with Tukey's comparison, or two-way mixed-model ANOVA followed by Bonferroni post hoc comparisons as appropriate. Differences were considered significant when $p<$ 0.05 .

\section{Results}

Hippocampal neuronal excitability and evoked synaptic transmission are unaltered in MKP-2 ${ }^{-/-}$mice Action potential firing in response to current injection (Fig. $1 a-c$ ), resting membrane potential $\left(\mathrm{MKP}-2^{+/+}:-68 \pm 1\right.$ $\mathrm{mV}, n=5$; MKP-2 $2^{-1-}:-67 \pm 1 \mathrm{mV}, n=$ $6)$ and input resistance (MKP-2 ${ }^{+/+}$: $131 \pm 6 \mathrm{M} \Omega, n=5$; MKP-2 $2^{-/-}: 130 \pm 5$ $\mathrm{m} \Omega, n=6$ ) were unaltered in slices from MKP-2 ${ }^{-1-}$ mice compared with MKP$2^{+/+}$controls. Similarly, evoked synaptic transmission was unaffected by MKP-2 deletion (Fig. 1d) as was the modulation of synaptic transmission by MAPK pathway inhibitors. Indeed, U0126 (40 $\mu \mathrm{M})$ and SP600125 $(20 \mu \mathrm{M})$, inhibitors of ERK and JNK, respectively, were without effect on basal synaptic transmission in both MKP-2 ${ }^{+/+}$and MKP-2 $2^{-1-}$ slices, whereas the p38 MAPK inhibitor, SB203580 (100 $\mu \mathrm{M})$ significantly increased basal synaptic transmission to a similar extent in MKP-2 $2^{+/+}$and MKP$2^{-1-}$ slices $\left(\mathrm{MKP}-2^{+/+}: 52 \pm 22 \%\right.$ increase, $n=8, \mathrm{MKP}^{-1-} 2^{-1} 51 \pm 8 \%$ increase, $n=5$ ).

\section{MKP-2 deletion results in increased sEPSC frequency}

With no effect of MKP-2 deletion on neuronal excitability and evoked synaptic transmission, we investigated whether MKP-2 played a role in modulating spontaneous neurotransmitter release. sEPSC frequency, but not amplitude, was significantly increased in $\mathrm{MKP}-2^{-1-}$ hippocampal slices compared with MKP- $2^{+/+}(p<0.05$; Fig. $1 e-h)$. This was also present in primary hippocampal cultures prepared from $\mathrm{MKP}-2^{+/+}$and MKP-2 $2^{-I-}$ mice. At $11 \mathrm{~d}$ in vitro (DIV), the frequency of sEPSCs, but not amplitude was significantly increased in MKP-2 ${ }^{-1-}$ cultures compared with MKP-2 $2^{+/+}$cultures $(p<0.05$; Fig. $2 a-d)$. Similarly, at $7 \mathrm{DIV}$, when little evidence of synaptic activity was observed in MKP-2 $2^{+/+}$cultures, the frequency of sEPSCs was significantly increased in MKP-2 $2^{-1-}$ cultures with no difference in sEPSC amplitude detected ( $p<0.05$; Fig. $2 e, f)$.

\section{Impaired LTP but normal PPF in MKP-2 $2^{-1-}$ mice}

Having investigated synaptic transmission, we next examined the role of MKP-2 in modulating hippocampal short-term and longterm plasticity. PPF (50-500 ms ISI) was unchanged between MKP-2 ${ }^{+/+}$and MKP-2 $2^{-1-}$ slices (Fig. $2 g$ ). Similarly, short-term potentiation was unchanged following LTP induction $(p>0.05$; Fig. $2 h$ ), whereas there was a significant impairment in the maintenance of LTP, with EPSC amplitude returning to baseline within 30 min of induction in MKP-2 $2^{-1-}$ slices while LTP was maintained in MKP-2 ${ }^{+/+}$slices $(p<0.01$; Fig. $2 h)$. b

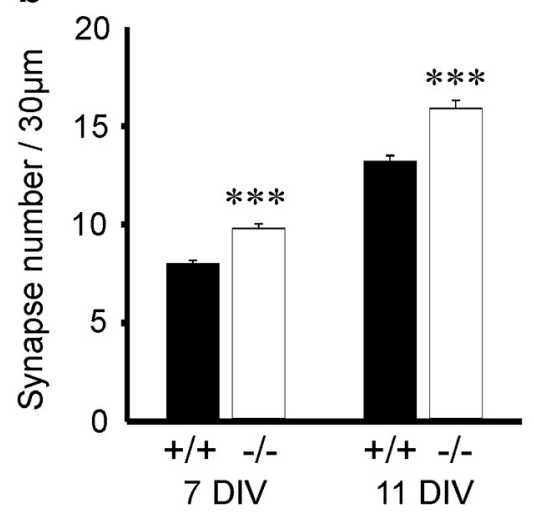

d

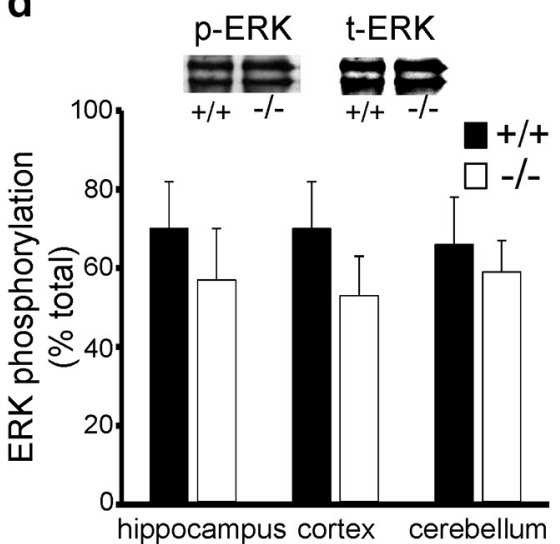

Figure 3. Increased synapse number in MKP-2 $2^{-/-}$hippocampal cultures. $\boldsymbol{a}$, Representative images and (b) bar chart summarizing the increased synapse number in MKP-2 $2^{-1-}$ hippocampal cultures. $c$, Hippocampal structure and (d) ERK activity is unaltered in MKP-2 $2^{-1-}$ mice. ${ }^{* *} p<0.001$. Scale bars: a, $10 \mu \mathrm{m} ; \mathbf{c}, 100 \mu \mathrm{m}$.

MKP-2 deletion increases synapse number but ERK activity is unaffected

An interpretation of our sEPSC data are a change in presynaptic function but no change in PPF suggests that probability of release in unchanged. An alternative explanation for increased sEPSC frequency is an increased synapse number. Hence, we investigated synapse number using the synaptic marker, synaptophysin in 7 and 11 DIV cultures. Synapse number was significantly increased at both 7 and 11 DIV in MKP-2 ${ }^{-1-}$ cultures compared with MKP- $2^{+/+}$cultures $(p<0.001$; Fig. $3 a, b)$. We further examined whether hippocampal structure or altered ERK activity contributed to this observation. Brain size and hippocampal structure were unaltered in $\mathrm{MKP}-2^{-1-}$ mice compared with MKP-2 ${ }^{+/+}$controls when examined using immunolabeling for $\mathrm{NeuN}$ (Fig. 3c). In addition, there were no significant differences in phospho-ERK levels in the hippocampus, the cortex, or the cerebellum (Fig. $3 d$ ), respectively, with no change in total ERK being observed in any of these brain regions. In addition, no change in ERK activity was seen at both 7 DIV (MKP-2 ${ }^{+/+}: 69 \pm$ $5 \%$ of total ERK, MKP-2 $2^{-1-}: 75 \pm 6 \%$ of total ERK, $\left.p>0.05\right)$ and 11 DIV cultures (MKP- $2^{+/+}: 86 \pm 7 \%$ of total ERK, MKP$2^{-1-}: 91 \pm 4 \%$ of total ERK, $\left.p>0.05\right)$.

\section{Impaired spatial reference and working memory in}

MKP-2 ${ }^{-1-}$ mice

Having established alterations in hippocampal synaptic plasticity, we probed hippocampal-dependent memory processes of MKP-2 ${ }^{-1-}$ mice using the MWM. Although MKP-2 ${ }^{-1-}$ mice revealed no changes in the OFT or EPM (Fig. $4 a, b$ ), on the first 
a

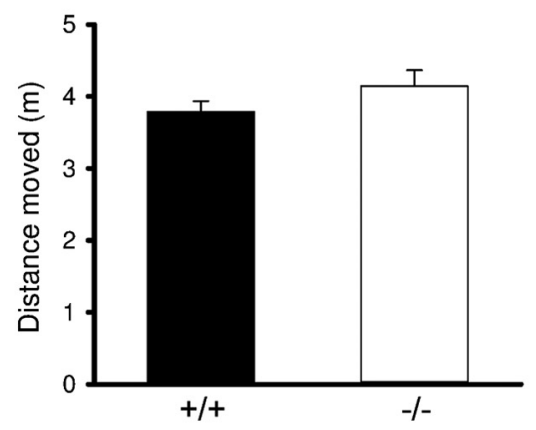

C

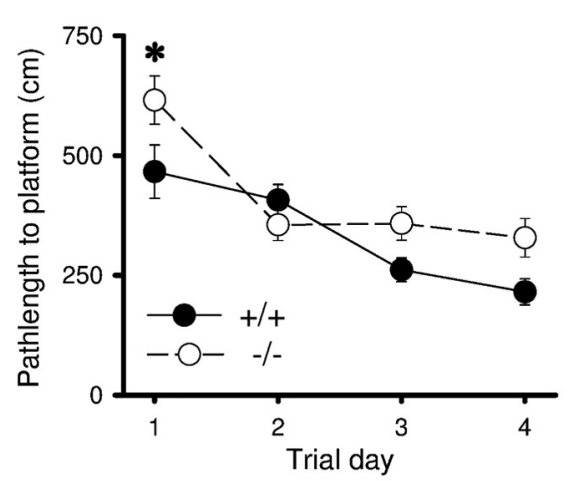

e

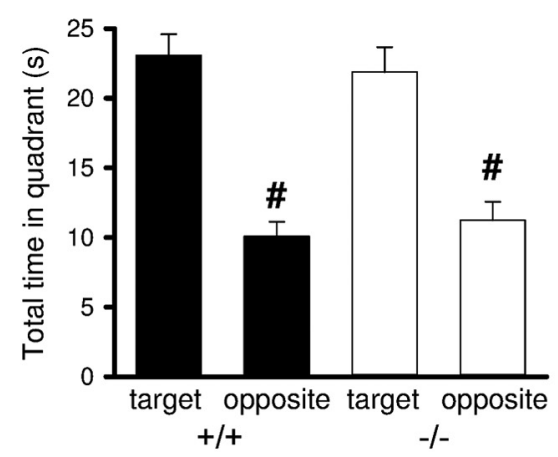

b

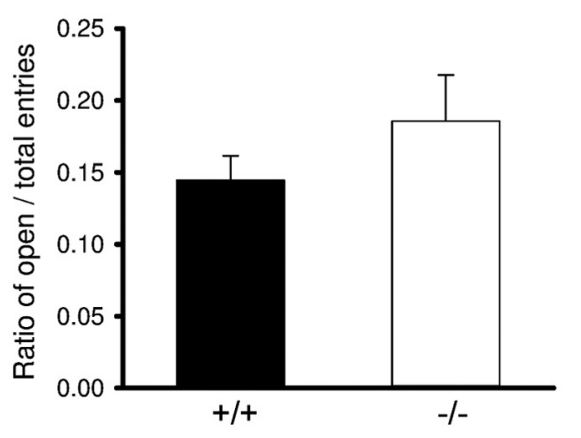

d

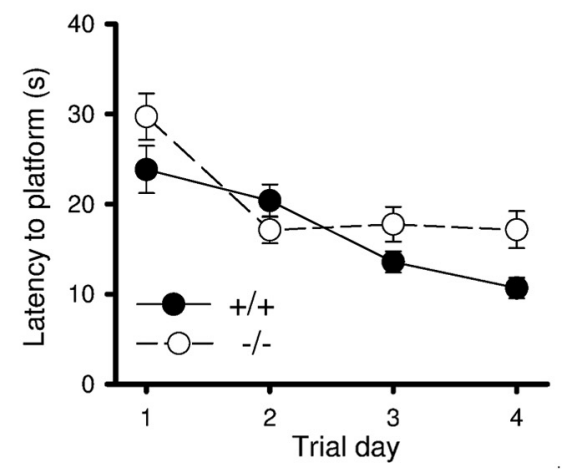

f

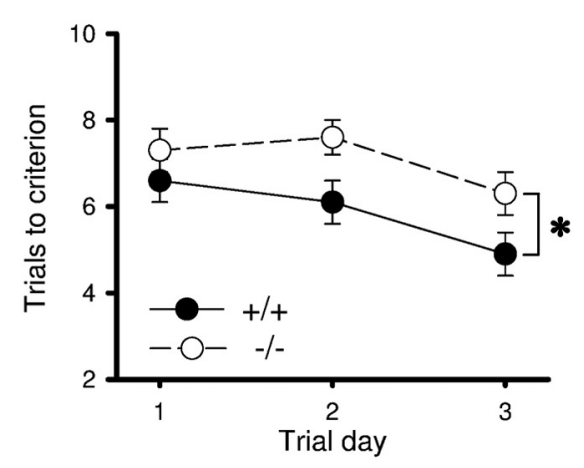

Figure 4. Impaired spatial reference and working memory in MKP-2 $2^{-1-}$ mice. $\boldsymbol{a}$, General locomotor activity is unaltered in MKP-2 $2^{-1-}$ mice as measured in the OFT. $\boldsymbol{b}$, Anxiety-like behavior is normal in MKP-2 $2^{-1-}$ mice as measured in the EPM. $\boldsymbol{c}$, Path-length to platform is significantly longer in MKP-2 $2^{-1-}$ mice, whereas (d) latency to the hidden platform in MWM reference memory paradigm is unchanged. $\boldsymbol{e}$, Both genotypes learnt the location of the platform over $4 \mathrm{~d}$ of the MWM $(\boldsymbol{f})$ trials to criterion is significantly higher in MKP-2 $2^{-1-}$ mice over 3 consecutive days of a MWM working memory paradigm. ${ }^{*} p<0.05$ versus MKP $-2^{+1+}$ controls; \#p $<0.05$ target versus opposite quadrant.

day of testing, the path-length to platform was significantly increased in MKP- $2^{-1-}$ mice $(p<0.05$; Fig. $4 c)$. However, on subsequent trial days (days $2-4$ ) there was no significant difference between the two genotypes. Furthermore, although all animals learnt the position of the platform, as evidenced by a significant decrease in path-length to locate the platform and latency to platform (Fig. $4 c, d$ ), and by the observation that all mice spent significantly more time in the target quadrant than in the opposite quadrant during the probe trial (Fig. 4e), this was not significantly different between the two genotypes. However, in a subsequent $3 \mathrm{~d}$ working memory paradigm, MKP- $2^{-1-}$ mice required significantly more trials to achieve criterion $(p<0.05$; Fig. $4 f)$ than MKP-2 ${ }^{+/+}$mice.

\section{Discussion}

In the absence of specific MKP-2 inhibitors, we have used MKP- $2^{-1-}$ mice to probe the function of MKP-2 in the CNS. We have shown that MKP-2 deletion results in increased sEPSC frequency and impaired hippocampal LTP in the absence of any deleterious effect on neuronal excitability, evoked synaptic transmission, or short-term plasticity. Furthermore, an increased synapse number was observed but no alternation in ERK activity was evident. Finally, whereas locomotor activity and anxiety-like behavior are unaltered in $\mathrm{MKP}-2^{-1-}$ mice, hippocampal-dependent memory processes were impaired.

A significant increase in sEPSC frequency, but not amplitude, with no overt effect on evoked synaptic transmission or PPF implies that vesicle release probability is unaffected as alterations would be expected in both sEPSC frequency and PPF if this were involved. MAPKs modulate release machinery and consequently neurotransmitter release with for example, ERK-dependent phosphorylation of synapsin 1 leading to an increase in docked vesicles, mEPSC frequency and PPF (Kushner et al., 2005; Giachello et al., 2010). The increase in sEPSCs we observe is consistent with these findings. However, given the observed lack of effect on PPF and ERK activity, it may be an alternative mechanism entirely that is involved. Indeed, an increase in synapse number in hippocampal MKP-2 $2^{-1-}$ cultures was seen that could account for the increased sEPSC frequency. This is in agreement with previous studies which have revealed that neurotransmitter release modulation but not PPF is a result of synapse number rather than changes in release probability (McClelland et al., 2010; Guimond et al., 2014). However, it should be noted that we examined only spontaneous excitatory neurotransmitter release whereas synaptophysin identifies all synapses so this increase in number may also include an increase in inhibitory synapses, hence we cannot rule out a role for MKP-2 in inhibitory transmission. Similarly, although we focused on ERK activity in the present study, this does not rule out a role of other MAPKs. However, whether an increase in synapse number alone underlies our observations would require examination of other potential mechanisms including localized presynaptic changes in MAPK activity and/or an increase in the number of docked vesicles. Despite no change in short-term plasticity, LTP was impaired in MKP- $2^{-1-}$ slices. The requirement for ERK in the induction and maintenance of LTP in mouse brain slices is dependent on the induction protocol used with previous studies indicating that the protocol used here would be ERK independent (Sweatt, 2004). 
Given that ERK activity was unchanged in our preparations, this may indicate that ERK plays no role in the impaired LTP observed here but whether changes in LTP would be observed using alternative ERK-dependent induction protocols remains unclear. However, a link between spontaneous neurotransmitter release and synaptic plasticity may indicate that the increased sEPSC plays a role in the impaired LTP seen here. Spontaneous neurotransmitter release is regulated by various neuromodulators (Kavalali, 2015) but recent studies indicate it also plays a critical role in synaptic scaling. Indeed, it is proposed that spontaneous neurotransmitter release regulates the expression of postsynaptic NMDA receptor subunit composition (Lee et al., 2010) and selective inhibition of spontaneous NMDA-mediated neurotransmission potentiates hippocampal synaptic responses (Nosyreva et al., 2013). Hence, given the increased sEPSC frequency observed in the present study, this implies that this increased synaptic activity reduces the ability of these synapses to exhibit LTP. However, whether changes in NMDA receptor subunit composition account for this observation is beyond the scope of this study but our data indicates it warrants further investigation using, for example, NMDA/AMPA ratio analyses.

As LTP is proposed to be a molecular correlate of memory, our data indicating deficits in hippocampal-dependent memory processes in $\mathrm{MKP}-2^{-1-}$ mice supports this view. Numerous studies have reported that both genetic and pharmacologic manipulation of hippocampal activity results in impaired LTP in vitro and performance in hippocampal-dependent memory processes in vivo but as to whether one does indeed underlie the other remains an area of intense debate (Takeuchi et al., 2013). However, our data revealing impaired spatial reference memory on the initial trial day and reduced performance in a subsequent working memory paradigm strongly indicates that the observed changes in synaptic function do indeed correlate with the observed behavioral deficits. In addition, it would be interesting to extrapolate these hippocampal findings to determine whether other brain regions associated with working memory including the prefrontal cortex, also exhibit similar alterations in synaptic activity and plasticity.

Together, these novel data indicate that MKP-2 plays a significant role in hippocampal synaptic function, with a particular locus being spontaneous rather than evoked neurotransmitter release. Further studies are required to fully examine the role of MKP-2 in normal brain functioning and in CNS disorders, particularly those in which memory deficits are prominent.

\section{References}

Al-Mutairi MS, Cadalbert LC, McGachy HA, Shweash M, Schroeder J, Kurnik M, Sloss CM, Bryant CE, Alexander J, Plevin R (2010) MAP kinase phosphatase-2 plays a critical role in response to infection by Leishmania mexicana. PLoS Pathog 6:e1001192. CrossRef Medline

Caunt CJ, Keyse SM (2013) Dual-specificity MAP kinase phosphatases (MKPs): shaping the outcome of MAP kinase signalling. FEBS J 280: 489-504. CrossRef Medline

Ciccarelli A, Giustetto M (2014) Role of ERK signaling in activity- dependent modifications of histone proteins. Neuropharmacology 80 : 34-44. CrossRef Medline

Cuevas BD, Abell AN, Johnson GL (2007) Role of mitogen-activated protein kinase kinase kinases in signal integration. Oncogene 26:3159-3171. CrossRef Medline

Duric V, Banasr M, Licznerski P, Schmidt HD, Stockmeier CA, Simen AA, Newton SS, Duman RS (2010) A negative regulator of MAP kinase causes depressive behavior. Nat Med 16:1328-1332. CrossRef Medline

Gan J, Greenwood SM, Cobb SR, Bushell TJ (2011) Indirect modulation of neuronal excitability and synaptic transmission in the hippocampus by activation of proteinase-activated receptor-2. Br J Pharmacol 163: 984-994. CrossRef Medline

Giachello CN, Fiumara F, Giacomini C, Corradi A, Milanese C, Ghirardi M, Benfenati F, Montarolo PG (2010) MAPK/Erk-dependent phosphorylation of synapsin mediates formation of functional synapses and shortterm homosynaptic plasticity. J Cell Sci 123:881-893. CrossRef Medline

Guimond D, Diabira D, Porcher C, Bader F, Ferrand N, Zhu M, Appleyard SM, Wayman GA, Gaiarsa JL (2014) Leptin potentiates GABAergic synaptic transmission in the developing rodent hippocampus. Front Cell Neurosci 8:235. CrossRef Medline

Jeanneteau F, Deinhardt K, Miyoshi G, Bennett AM, Chao MV (2010) The MAP kinase phosphatase MKP-1 regulates BDNF-induced axon branching. Nat Neurosci 13:1373-1379. CrossRef Medline

Kavalali ET (2015) The mechanisms and functions of spontaneous neurotransmitter release. Nat Rev Neurosci 16:5-16. CrossRef Medline

Keshet Y, Seger R (2010) The MAP kinase signaling cascades: a system of hundreds of components regulates a diverse array of physiological functions. Methods Mol Biol 661:3-38. CrossRef Medline

Krementsov DN, Thornton TM, Teuscher C, Rincon M (2013) The emerging role of $\mathrm{p} 38$ mitogen-activated protein kinase in multiple sclerosis and its models. Mol Cell Biol 33:3728-3734. CrossRef Medline

Kushner SA, Elgersma Y, Murphy GG, Jaarsma D, van Woerden GM, Hojjati MR, Cui Y, LeBoutillier JC, Marrone DF, Choi ES, De Zeeuw CI, Petit TL, Pozzo-Miller L, Silva AJ (2005) Modulation of presynaptic plasticity and learning by the $\mathrm{H}$-ras/extracellular signal-regulated kinase/synapsin I signaling pathway. J Neurosci 25:9721-9734. CrossRef Medline

Lawan A, Torrance E, Al-Harthi S, Shweash M, Alnasser S, Neamatallah T, Schroeder J, Plevin R (2012) MKP-2: out of the DUSP-bin and back into the limelight. Biochem Soc Trans 40:235-239. CrossRef Medline

Lee MC, Yasuda R, Ehlers MD (2010) Metaplasticity at single glutamatergic synapses. Neuron 66:859-870. CrossRef Medline

McClelland AC, Hruska M, Coenen AJ, Henkemeyer M, Dalva MB (2010) Trans-synaptic EphB2-ephrin-B3 interaction regulates excitatory synapse density by inhibition of postsynaptic MAPK signaling. Proc Natl Acad Sci U S A 107:8830-8835. CrossRef Medline

Nosyreva E, Szabla K, Autry AE, Ryazanov AG, Monteggia LM, Kavalali ET (2013) Acute suppression of spontaneous neurotransmission drives synaptic potentiation. J Neurosci 33:6990-7002. CrossRef Medline

Samuels IS, Saitta SC, Landreth GE (2009) MAP'ing CNS development and cognition: an ERKsome process. Neuron 61:160-167. CrossRef Medline

Sweatt JD (2004) Mitogen-activated protein kinases in synaptic plasticity and memory. Curr Opin Neurobiol 14:311-317. CrossRef Medline

Takeuchi T, Duszkiewicz AJ, Morris RG (2013) The synaptic plasticity and memory hypothesis: encoding, storage and persistence. Philos Trans R Soc Lond B Biol Sci 369:20130288. CrossRef Medline

Thomas GM, Huganir RL (2004) MAPK cascade signalling and synaptic plasticity. Nat Rev Neurosci 5:173-183. CrossRef Medline

Wancket LM, Frazier WJ, Liu Y (2012) Mitogen-activated protein kinase phosphatase (MKP)-1 in immunology, physiology, and disease. Life Sci 90:237-248. CrossRef Medline

Yasuda S, Sugiura H, Tanaka H, Takigami S, Yamagata K (2011) p38 MAP kinase inhibitors as potential therapeutic drugs for neural diseases. Cent Nerv Syst Agents Med Chem 11:45-59. CrossRef Medline 